\title{
Correction to: Influence of community-based natural resource management strategies in the resilience of social-ecological systems
}

\author{
Maria del Mar Delgado-Serrano ${ }^{1}$ - Elisa Oteros-Rozas ${ }^{2,3}$ • Isabel Ruiz-Mallén ${ }^{4,5}$. \\ Diana Calvo-Boyero ${ }^{5}$ - Cesar Enrique Ortiz-Guerrero ${ }^{6}$. \\ Roberto Ivan Escalante-Semerena ${ }^{7} \cdot$ Esteve Corbera $^{5}$
}

Published online: 23 October 2017

(C) Springer-Verlag GmbH Germany 2017

Correction to: Reg Environ Change (2017)

https://doi.org/10.1007/s10113-017-1223-4

The published online version contains mistake. Author name was incorrectly captured. Instead of Maria del Mar Delgado-Serrano was incorrectly captured as Ma del Mar Delgado-Serrano. Correct author group section is shown above.

The original article was corrected.

The online version of the original article can be found at https://oi.org/ 10.1007/s10113-017-1223-4

Maria del Mar Delgado-Serrano

mmdelgado@uco.es

1 Department of Agricultural Economics, Universidad de Córdoba, Campus de Rabanales, C5, planta 3, 14071 Córdoba, Spain

2 Social and Participatory Action Research Group, Universidad Pablo de Olavide, Sevilla, Spain

3 Department of Geosciences and Natural Resource Management, University of Copenhagen, Copenhagen, Denmark

4 Internet Interdisciplinary Institute (IN3), Universitat Oberta de Catalunya, Barcelona, Spain

5 Institute of Environmental Science and Technology, Universitat Autònoma de Barcelona, Barcelona, Spain

6 Pontificia Universidad Javeriana, Bogotá, Colombia

7 Universidad Nacional Autónoma de Mexico, Mexico City, Mexico 